\title{
Diffraction of electromagnetic waves on the resonance graphene metasurfaces
}

\author{
Alexander M. Lerer ${ }^{1, *}$ and Galina S. Makeeva ${ }^{2}$ \\ ${ }^{1}$ Southern Federal University, Faculty of Physics, 344090 Rostov-on-Don, Russia \\ ${ }^{2}$ Penza State University, Department of Radioengineering, 440026 Penza, Russia
}

\begin{abstract}
By using three independent numerical approaches to solve the 3D diffraction boundary problems the reflection, transmittance coefficients and losses of metasurfaces of graphene strip elements, depending on the frequency and values of the chemical potential were calculated in the terahertz frequency range. The results of numerical simulation of performances of tuned by the external bias electric field $\mathrm{THz}$ absorbers and $\mathrm{THz}$ polarizers based on the resonance metasurfaces of single square and two connected rectangular graphene strips in the unit cell placed on the dielectric or multilayer graphene-dielectric substrates were obtained.
\end{abstract}

\section{Introduction}

The combination of graphene and metasurfaces presents a powerful tunability for exotic electromagnetic properties, where the metasurfaces with the highly-localized fields offer a platform to enhance the interaction between the incident waves and graphene and facilitate a deep modulation [1].

A new approach for design of ultra-wideband $\mathrm{THz}$ absorber with polarization and incident angle insensitivity was proposed using metal and graphene hybrid metasurfaces [2] and the robust design method for extremely broadband, wide-angle, and polarizationinsensitive $\mathrm{THz}$ absorbers developed using multilayer graphene-based metasurfaces [3]:

Novel terahertz (THz) absorbers made up of periodic single- and double-layer decussate graphene ribbon arrays have narrowband near-unity $\mathrm{THz}$ absorption with ultra-wide frequency reconfiguration and angular stability [4].

Ultrathin metasurfaces based on arrays of L-shaped graphene periodic patches were demonstrated to achieve broadband cross-polarization transformation in reflection and transmission [5]. These metasurface designs have tunable responses and are envisioned to become the building blocks of several integrated $\mathrm{THz}$ systems [5].

The development and manufacturing of graphene-based materials and integrated plasmonic devices depend on the development of simulation tools, based on accuracy and the adequacy of mathematical models by solving Maxwell's equations rigorously.

The goal of the present work is to study diffraction phenomena, geometry and size effects and to design of $\mathrm{THz}$ absorbers and polarizers based on the resonance metasurfaces of graphene strip elements using rigorous mathematical models to solve the $3 \mathrm{D}$ diffraction boundary problems.

\footnotetext{
*Corresponding author: lerer@sfedu.ru
} 


\section{Mathematical models and methods}

The mathematical models of diffraction and interactions of electromagnetic waves with the resonance graphene-based metasurfaces are based on the solution of diffraction 3D boundary problems for the Maxwell's equations with electrodynamic boundary conditions, simultaneously with a model of graphene surface conductivity as a Kubo formalism [6].

The simulation of absorbers involves two specific methods. In the first method of the approximate boundary conditions $(\mathrm{ABCs})$, graphene layers are substituted with infinitely thin impedance layers, whose impedance is determined by the Kubo formula. In this approach, a task is reduced to the solution of paired summation equations relative to the current density on the bands [7]. The equations can be solved via the Galerkin method, where the basic functions are the Gegenbauer polynomials. The second method underlies the numerically analytical method for solving a volume integrodifferential equation (VIDE) for the dielectric bodies [8].

The electrodynamic model of polarizers is developed to solve the diffraction 3D boundary problem using the decomposition approach [9] on autonomous blocks with Floquet channels (FABs) [9].

\section{Results of numerical modeling of $\mathrm{THz}$ absorbers based on resonance metasurfaces of graphene strips elements on the dielectric or multilayer graphene-dielectric substrates}

Let's assume that a monochromatic, homogeneous plane electromagnetic wave (TEMwave, fields $\boldsymbol{E}, \boldsymbol{H}$; wave vector $\boldsymbol{k}$, frequency $\omega$ ) is incident ( $\varphi-$ angle of orientation of the electric field $\boldsymbol{E}$ of incident TEM-wave respective to graphene strip) on the metasurface of graphene strip elements ( $a, b$ - periodicity along axes $x, y ; w$-wide, $l$ - length of graphene strips), on the dielectric (or multilayer graphene-dielectric) substrate, $h$ - thickness (Fig.1).

The frequency dependences calculated for resonance metasurfaces of graphene strip elements are shown in Fig. 2,3,5,6. The electrodynamic calculations are performed assuming the normal incidence of the $T E M$-wave (for angle of incidence $\theta=0$ ).

The results of calculation of the reflection $R=\left|\mathrm{S}_{11}\right|^{2}$, transmittance $T=\left|\mathrm{S}_{21}\right|^{2}$ coefficients and losses $P=1-R-T$ in the metasurface of single square graphene strip in the unit cell placed on the dielectric substrate for different values of chemical potential $\mu_{c}$ are shown in fig. 2 .

The results of modelling of the metasurfaces of two connected identical rectangular graphene strips in the unit cell on the dielectric substrate are shown in fig. 3. These rectangular graphene strips are connected on the wide side (Fig.1).

In the case $s$-polarization (the orientation of the electric field $\boldsymbol{E}$ of incident wave is parallel to the strips, $\varphi=0$ (Fig. $1 \mathrm{~b}$ )) the plasmon resonance is observed along the wide side of the graphene strip element, the resonance frequency is close to one of the metasurface of single strip in the unit cell (Fig. 2). This resonance frequency is weakly dependent on the size of gap $d$ (fig. $3 \mathrm{a}, \mathrm{b}$ ).

In the case of orthogonal $p$-polarization (the electric field $\boldsymbol{E}$ is normal to the strip, $\varphi=90^{\circ}$ (Fig.1b)) the plasmon resonance is observed along the narrow side of the graphene strip element (Fig.1b) and therefore the resonance frequencies increase (fig. 3). This plasmon resonance frequency depends on the size of the gap $d$ between strips (Fig.1b) and increases with decreasing the distance $d$ (Fig. 3a, b). At small distances $(d=1 \mu \mathrm{m})$ between the graphene strips a low-frequency resonance appears (Fig. 3.a), which is close in to the frequency resonance for $s$-polarization. 


\section{Resonance metasurface of graphene strip elements}

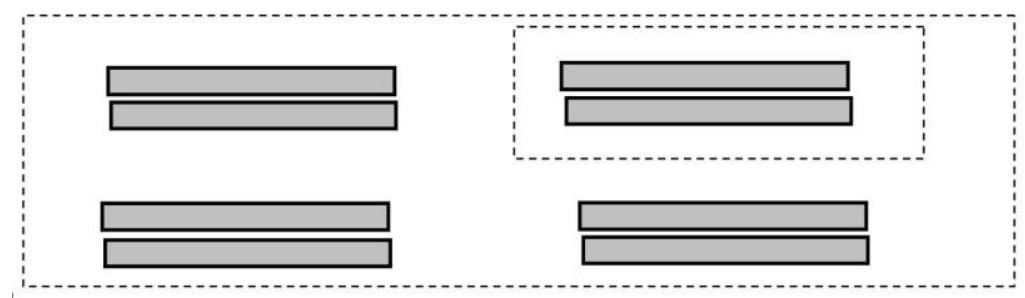

a)

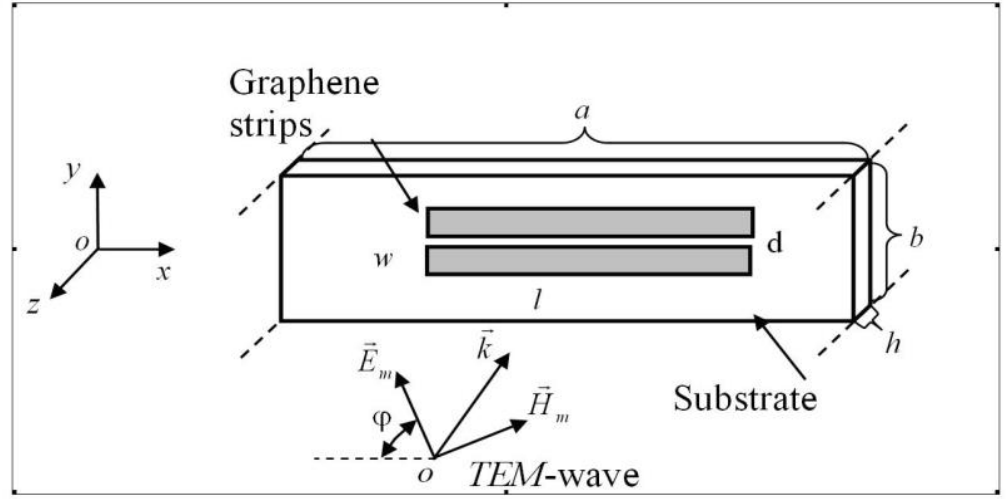

b)

Fig. 1. Geometry of metasurface for the diffraction problem: a) periodic graphene structure on the dielectric (or multilayer) substrate, b) model of the unit cell of the array; direction of incident TEMwave of wave vector $\boldsymbol{k} ; \varphi$ - angle of orientation of the electric field $\boldsymbol{E}$ respective to graphene strip; periodicity $a, b ; w$-width, $l$-length of graphene strip; $d$-distance between strips; $h$-thickness of substrate.

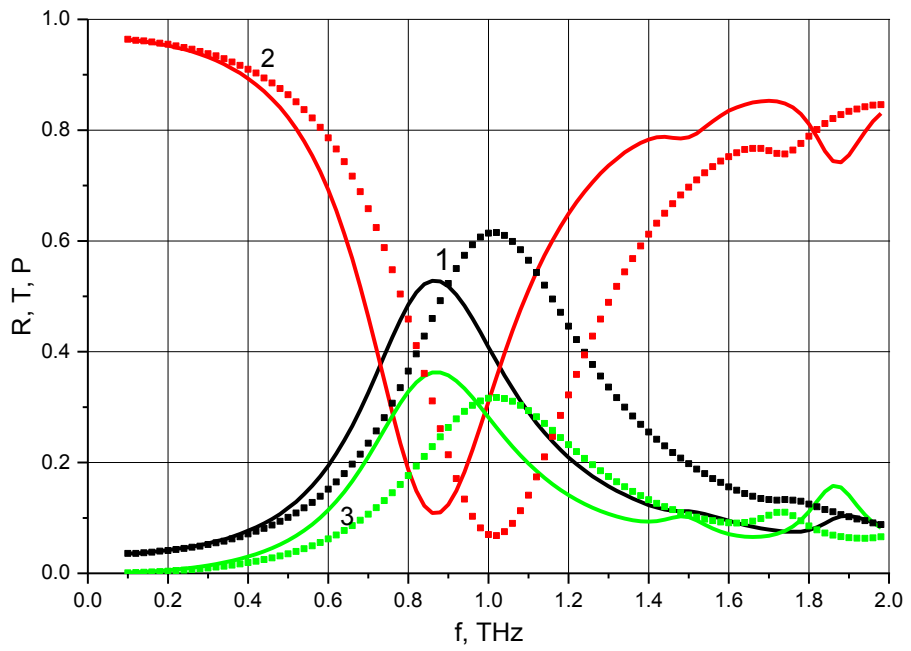

Fig. 2. Frequency dependences of the reflection, transmittance coefficients and losses in the resonance metasurface of single square graphene strip in the unit cell on the dielectric substrate: Curves $1-R$, $2-T, 3-P ; W=l=50 \mu \mathrm{m}, \mathrm{a}=\mathrm{b}=70 \mu \mathrm{m} ; h=0.55 \mu \mathrm{m} ; \mathrm{n}=1.45$ : Parameters of graphene are $T=300 \mathrm{~K}$, and $\tau=1$ ps. Solid lines $\mu_{c}=0.25 \mathrm{eV}$; dashed lines $\mu_{c}=0.35 \mathrm{eV}$. 


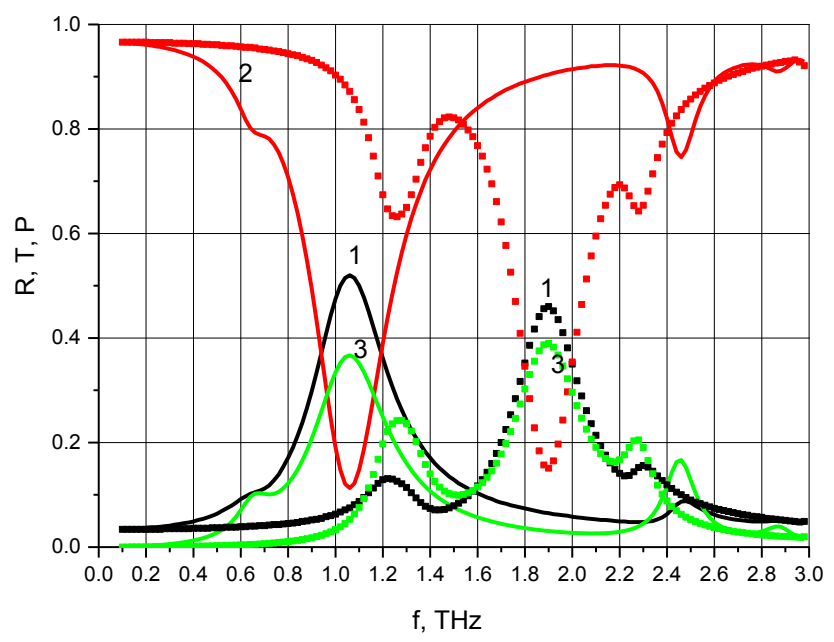

a)

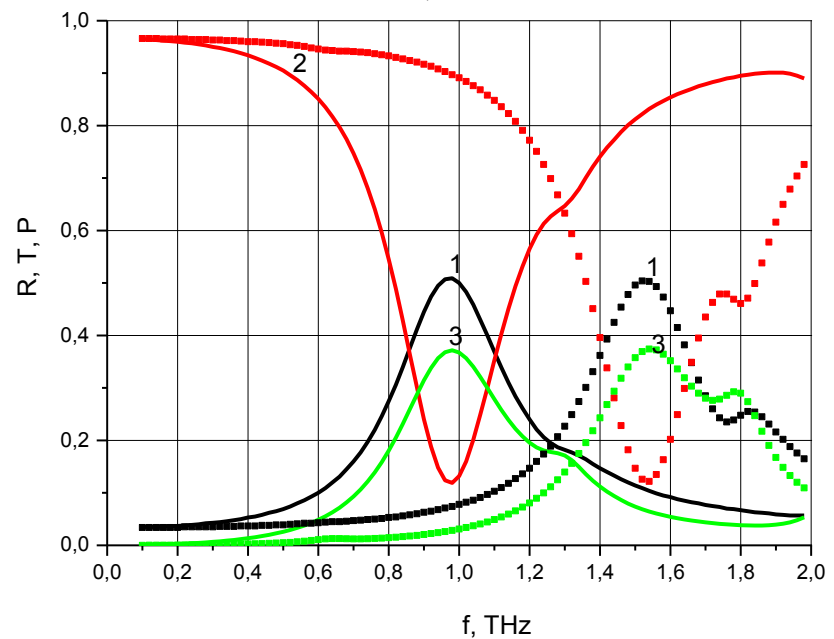

b)

Fig. 3. Frequency dependences of the reflection, transmittance coefficients and losses in the resonance metasurface of two connected identical rectangular graphene strips in the unit cell on the dielectric substrate: a) $d=1 \mu \mathrm{m}$; b) $d=10 \mu \mathrm{m}$; curves $1-R, 2-T, 3-P$; $W_{l}=W_{2}=25 \mu \mathrm{m}, l_{l}=l_{2}=50 \mu \mathrm{m} ; \mu_{c}=$ $0.25 \mathrm{eV}$. Solid lines s-polarization, dashed lines $p$-polarization of incident TEM-wave. Other parameters are as in Fig. 2.

Fig. 4 shows the frequency dependences of the current density (the current flowing along the direction of the electric field $\boldsymbol{E}$ of the incident $T E M$-wave) in the arrays of single or two connected graphene strips in the unit cell on the dielectric substrate.

The amplitude of current in the perpendicular direction is several orders of magnitude smaller. To estimate the average current density, the current strength must be divided by the width of the strip in the direction perpendicular to the current. Curves are normalized to a maximum of curve 5 .

From fig. 4 follows that in the case of $p$-polarization the current density and field intensity in connected graphene strips at resonance is almost two times higher than the current density in the array of single strips. When reducing the size $d$ of gap the density, and hence the intensity of the near-field increases. An even greater increase in current when connecting strips along the narrow side. 


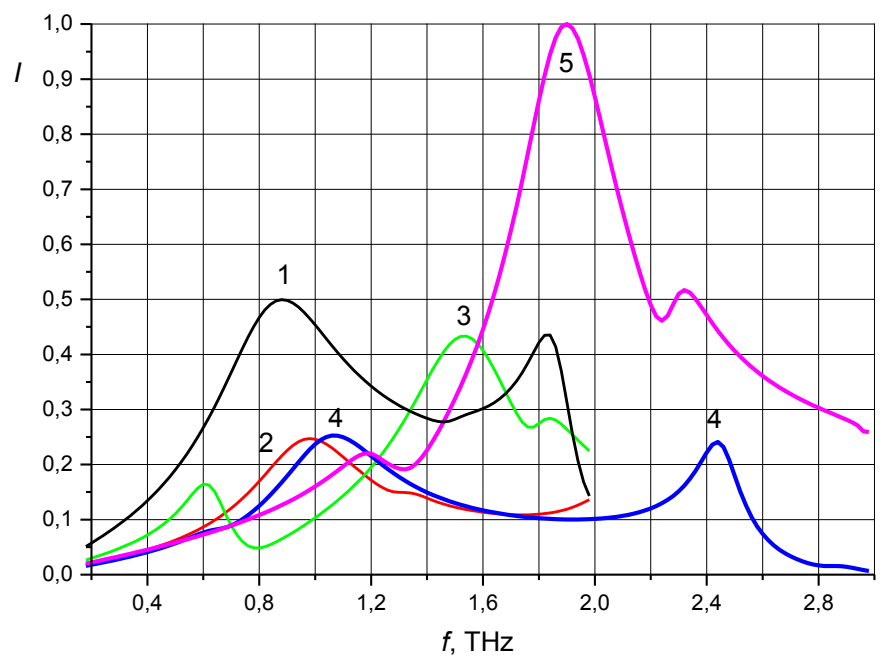

Fig. 4. Frequency dependences of the current density in the arrays of single or two connected graphene strips in the unit cell on the dielectric substrate: curves 1 - single graphene strip; 2 - two connected identical rectangular graphene strips $W=25 \mu \mathrm{m}, l=50 \mu \mathrm{m}$. Curves 2 - $s$-polarization, $d=10 \mu \mathrm{m} ; 3$ - $p$ polarization, $\mathrm{d}=10 \mu \mathrm{m} ; 4$ - $s$-polarization, $d=1 \mu \mathrm{m} ; 5$ - $p$-polarization, $d=10 \mu \mathrm{m} ; \mu_{c}=0.25 \mathrm{eV}$. Other parameters are as in Fig. 2.

The results of calculation of the reflection, transmittance coefficients and losses of metasurfaces of single square graphene strip elements in the unit cell based on multilayered substrates of graphene and dielectric layers are shown in fig. 5, 6 .

As follows from the results the frequency of plasmon resonance of metasurfaces of single square graphene strips depends on the distance between strips. As the sizes of graphene strip elements and the periodicity of $2 \mathrm{D}$ array increases, the plasmon resonance frequency is changing and maximum of resonance absorption is increases (fig. 5, 6).

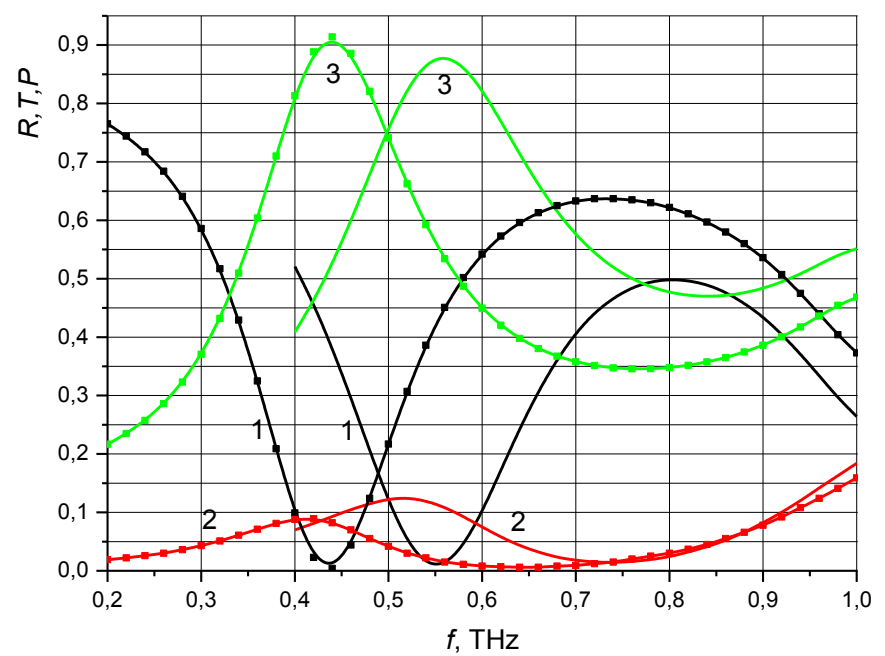

Fig. 5. Frequency dependences of the reflection, transmittance coefficients and losses in the resonance metasurfaces of single square graphene strip in the unit cell on the multilayer graphenedielectric substrate: Curves $1-R, 2-T, 3-P$; $\mathrm{a}=\mathrm{b}=70 \mu \mathrm{m}$. Seven -layer structure $h_{d}=25 \mu \mathrm{m}$, and $n_{d}=1.77: \mu_{\mathrm{c}}=0.25 \mathrm{eV}$. Lines with symbols $W=l=60 \mu \mathrm{m}$; lines without symbols $W=l=50 \mu \mathrm{m}$. Other parameters are as in Fig. 2. 


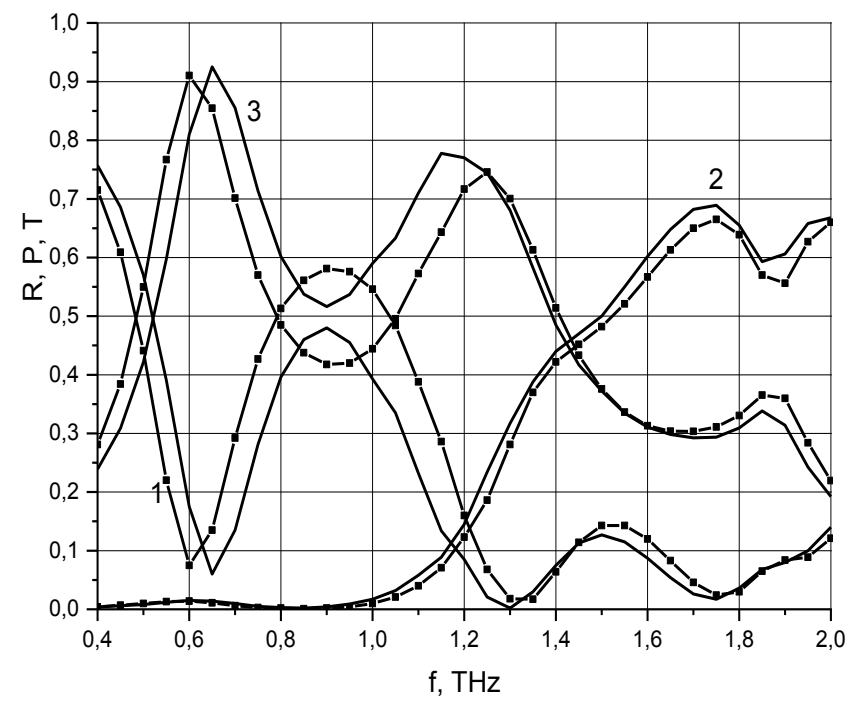

Fig. 6. Frequency dependences of the reflection, transmittance coefficients and losses in the resonance metasurfaces of single square graphene strip in the unit cell based on multilayered graphene-dielrectric substrate for different periodicity (density) of array : Curves $1-R, 2-T, 3-P . w=l=50 \mu \mathrm{m}, h_{d}=25$ $\mu \mathrm{m}$, and $n_{d}=1.77$. Nine-layer structure. Parameters of graphene are $T=300 \mathrm{~K}, \mu_{c}=0.25 \mathrm{eV}$, and $\tau=$ 1 ps. Lines with symbols are sizes of $a=b=70 \mu \mathrm{m}$; without symbols, $a=b=80 \mu \mathrm{m}$.

According to results of the numerical simulation, the metasurfaces of square graphene stripes on a multilayered substrates composed of graphene and dielectric layers are the effective absorbers in a $\mathrm{THz}$ frequency range, which absorbs almost $100 \%$ of the incident energy at the plasmon resonance frequency.

\section{Results of numerical simulation of THz polarizers based on resonance metasurfaces of rectangular graphene strips on the dielectric substrates}

By using the computational algorithm, based on the decomposition approach by FABs, the performances of $\mathrm{THz}$ polarizers, based on the resonance metasurfaces of graphene rectangular strips, depending on the value of chemical potential (the external bias electric field) were calculated at $\mathrm{THz}$ frequency range.

The results of calculation of the transmittance coefficient $\left|S_{21}\right|$ through $\mathrm{THz}$ polarizer based on the resonance metasurface of single rectangular graphene strip in the unit cell placed on the dielectric substrate (Fig.1) depending on the frequency for different angles $\varphi$ of orientation of the electric field $\boldsymbol{E}$ of incident TEM-wave respective to graphene strip (Fig.1b) are shown in Fig. 7.

Fig. 7 shows that upon changing the angle $\varphi$ of orientation of the electric field $\boldsymbol{E}$ of incident $T E M$-wave respective to graphene strip from $\varphi=0$ (the electric field $\boldsymbol{E}$ is parallel to the strips (Fig. 1 b)) to $\varphi=90^{\circ}$ (electric field $\boldsymbol{E}$ is normal to the strips) the value of the minima of the transmittance coefficient $\left|\mathrm{S}_{21}\right|$ decreases, however, its location is moving insignificantly. A stop band occurs around the resonant frequency due to plasmon resonance, for angle $\varphi=90^{\circ}$ the value of minimum of the transmittance coefficient $\left|\mathrm{S}_{21}\right|$ at the resonant frequency $f=28.7 \mathrm{THz}$ is equal to $22 \mathrm{~dB}$.

Fig. 8 shows the calculated dependence of the transmittance coefficient $\left|S_{21}\right|$ through $\mathrm{THz}$ polarizer on the angle of orientation of the electric field $\boldsymbol{E}$ of incident TEM-wave respective to graphene strip at the resonance frequency $f=28.7 \mathrm{THz}$ for $\mu_{c}=0.57 \mathrm{eV}$. 


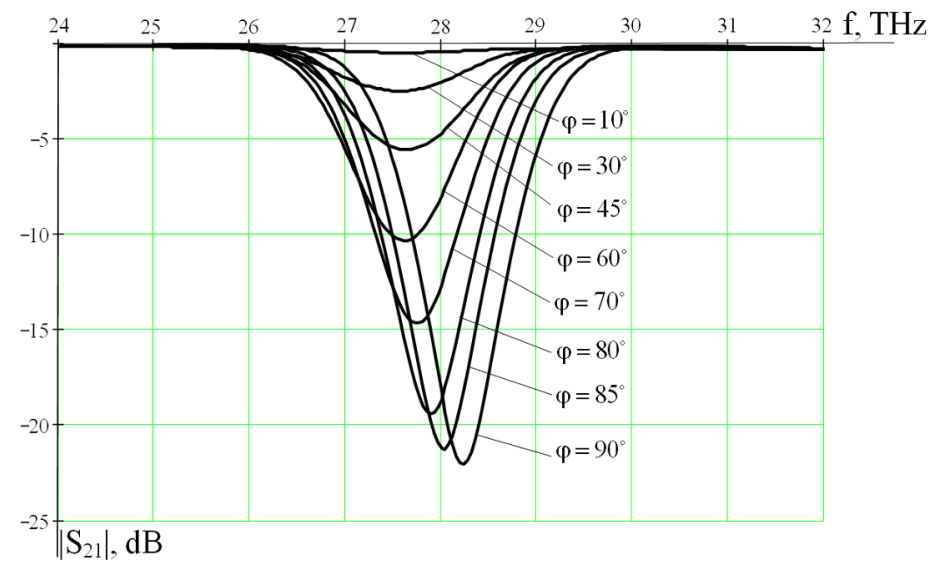

Fig. 7. Frequency dependences of transmittance coefficient $\left|S_{21}\right|$ through $\mathrm{THz}$ polarizer based on the resonance metasurface of single rectangular graphene strip in the unit cell on the dielectric substrate for different angles $\varphi$ of orientation of the electric field $\boldsymbol{E}$ of incident $T E M$-wave respective to graphene strip: $\mu_{c}=0.57 \mathrm{eV} ; a=2 \mu \mathrm{m}, b=0.2 \mu \mathrm{m}, w=0.1 \mu \mathrm{m}, l / a=0.9, h=0.5 \mu \mathrm{m}, \varepsilon=2.2$.

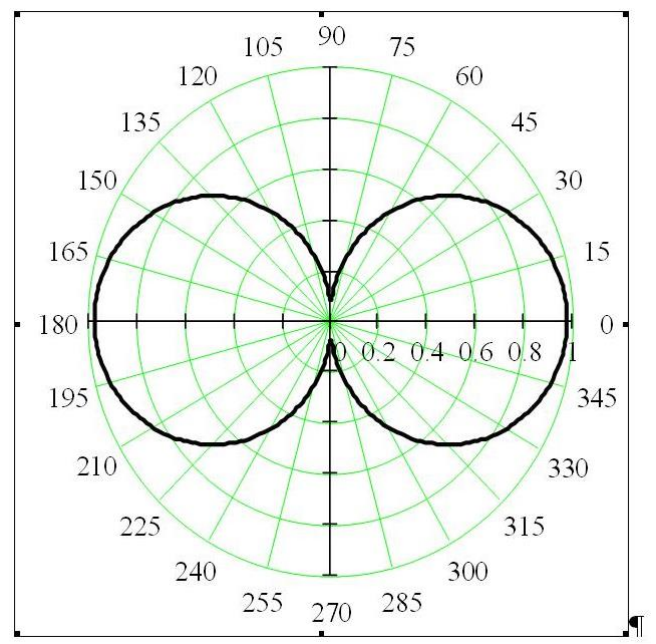

Fig. 8. Transmittance coefficient $\left|\mathrm{S}_{21}\right|$ through $\mathrm{THz}$ polarizer based on the resonance metasurface of single rectangular graphene strip in the unit cell on the dielectric substrate depending on the angle $\varphi$ of orientation of the electric field $\boldsymbol{E}$ of incident $T E M$-wave respective to graphene strip: $f=28.7 \mathrm{THz} ; \mu_{c}$ $=0.57 \mathrm{eV} ; a=2 \mu \mathrm{m}, b=0.2 \mu \mathrm{m}, w=0.1 \mu \mathrm{m}, l / a=0.9, h=0.5 \mu \mathrm{m}, \varepsilon=2.2$.

In the stop band the resonance metasurface of single rectangular graphene strip in the unit cell on the dielectric substrate can be considered as a THz polarizer [11].

\section{Conclusion}

The research has the potential to give insight into diffraction phenomena, related to novel and unique graphene-based metasurfaces and nanostructures, and to contribute to the development new integrated plasmonic $\mathrm{THz}$ devices.

The outcome of this work is the development of effective computational algorithms for solving diffraction boundary 3D problems for Maxwell's equations (with electrodynamic boundary conditions), simultaneously with a model of graphene surface conductivity as a Kubo formalism. 
It is shown that at the surface plasmon polariton resonant frequencies the metasurfaces of graphene strips on the dielectric or multilayer graphene-dielectric substrates are electrically tunable absorbers which absorbs almost $100 \%$ of the incident energy and effective polarizers at $\mathrm{THz}$ frequency range.

\section{Acknowledgment}

The work was supported as part of the basic part of a state task, project no. 3.5398.2017/8.9.

\section{References}

1. Ch. Liu, Y. Bai, $\dagger$ J. Zhou, Q. Zhao, and L. Qiao, A Review of Graphene Plasmons and its Combination with Metasurface, J. Korean Phys. Soc. 54 (5), 349 (2017)

2. L. Peng, X. Li M., X. Liu, X. Jiang and S. Lic. Metal and graphene hybrid metasurface designed ultra-wideband terahertz absorbers with polarization and incident angle insensitivity. Nanoscale Adv, 1, 1452 (2019)

3. M. Rahmanzadeh, H. Rajabalipanah and A. Abdolali. Multilayer graphene-based metasurfaces: robust design method for extremely broadband, wide-angle, and polarization-insensitive terahertz, Applied Optics, 57, 959 (2018)

4. L. Ye, F. Zeng, Y. Zhang, X. Xu, X. Yang, Q. Liu, Frequency-Reconfigurable WideAngle Terahertz Absorbers Using Single- and Double-Layer Decussate Graphene Ribbon Arrays. Nanomaterials, 8 (10), 834 (2018)

5. T. Guo, C. Argyropoulos, Broadband polarizers based on graphene metasurfaces. Optics Letters, 41 (23), 5592 (2016)

6. G.W. Hanson, Dyadic Green's functions and guided surface waves for a surface conductivity model of grapheme, J. Appl. Phys., 103, 064302 (2008)

7. A. M. Lerer and I. N. Ivanova, Application of approximate boundary conditions for calculation of planar 2D periodic nanoplasmon structures, J. Commun. Technol. Electron, 61 (5), 486 (2016)

8. A. M. Lerer, Theoretical investigation of $2 D$ periodic nanoplasmon structures, J. Commun. Technol. Electron., 57 (11), 1151 (2012)

9. 2. V.V. Nikol'skii and T.I. Nikol'skaya, Decomposition Approach in Electrodynamics Problems (Nauka, Moscow, 1983) [in Russian]

10. O. A. Golovanov. Solution of Applied Problems of Electromagnetics Based on the Use of Autonomous Blocks with Virtual Floquet Channels. J. Commun. Techn. Electron. 51, 1423 (2006)

11. A.M. Lerer, G.S. Makeeva, Polarization Effects and Resonance Absorption in Terahertz Wave Diffraction on Graphene Metasurfaces, Optics and Spectroscopy, 125 (6), 1034 (2018) 